\title{
Comparación de tres tecnologias en la preservación de conidias de hongos filamentosos y pruebas de viabilidad
}

Lydia Toscano Palomar ${ }^{1}$, Eric Vertiz Pelaez², Lourdes Cervantes Díaz ${ }^{3}$, Maria Guadalupe Amado Moreno ${ }^{4}$, Ángel García Velázquez ${ }^{5}$, Mary Triny Beleño-Cabarcas ${ }^{6}$ y Moisés Jesús Castro-Toscano ${ }^{7}$

\section{Resumen}

Conservar una colección de hongos es una tarea que demanda constante dedicación y vigilancia. Requiere conocer sus características morfológicas, fisiológicas y bioquímicas, así como sus requerimientos en cuanto a los métodos de preservación. El objetivo del presente trabajo consistió en evaluar la viabilidad, pureza y estabilidad morfológica de siete cepas de hongos filamentosos, previamente identificados. Las cepas identificadas como Aspergillus niger, Aspergillus awamori, Aspergillus oryzae, Aspergillus flavus, Penicillium chrysogenum, Penicillium sp., y Trichoderma harzianum, fueron subcultivadas y preservadas en placas con medio agar dextrosa papa (ADP) durante 18 meses. La preservación se realizó tratando las conidias obtenidas de los cultivos. Las conidias fueron sujetas a tres diferentes métodos de preservación: a) liofilización, b) en suelo-Arena y c) en papel filtro. Un mantenimiento a largo plazo es esencial para estudios detallados de las especies. La viabilidad y pureza de las especies fungales se monitorearon después de un almacenamiento a $10^{\circ} \mathrm{C}$ de 2 meses a dos años. En la preparación de la suspensión de conidias para liofilización se utilizaron Tritón X-100 como estabilizante y leche descremada como solución crioprotectora. El método de liofilización fue favorable pero destructible comparado con el método de secado en papel filtro dando como resultado daños severos al material fungal lo cual se reflejó en tiempos prolongados de germinación. El método de preservación en suelo-arena mostró las menores

1 Profesora Investigadora, Departamento de Ingeniería Química, Instituto Tecnológico de Mexicali, Mexico.

2 Tesista de Licenciatura, Instituto Tecnológico de Mexicali, México.

3 Instituto de Ciencias Agrícolas, Universidad Autonoma de Baja California, Mexico.

4 Departamento de Ciencias Básicas, Instituto Tecnologico de Mexicali, México.

5 Departamento de Ciencias Básicas, Instituto Tecnológico de Mexicali, Mexic.

6 Estudiante de Posgrado, Instituto de Ingeniería, Universidad Autónoma de Baja California.

7 Estudiante de Posgrado, Universidad Autónoma de Baja California, Facultad de Ingeniería. 
viabilidades de los métodos. Se concluye que el método de liofilización es un método simple y confiable para la preservación a largo plazo el cual presenta menos riesgos de contaminación.

Palabras clave: Viabilidad, preservación, liofilización y hongos filamentosos.

\section{Abstract}

Maintain and preserve a collection of fungi is a task that demands constant vigilance and dedication. It requires knowledge of the morphological, physiological and biochemical characteristics of fungi and their requirements in terms of preservation methods. The aim of this study was to assess the viability, purity and macroscopic and microscopic morphological stability of seven strains of filamentous fungi, previously identified in collaborative work by the Technological Institute of Mexicali and the Institute of Agricultural Sciences. The strains identified as Aspergillus niger, Aspergillus awamori, Aspergillus oryzae, Aspergillus flavus, Penicillium chrysogenum, Penicillium sp. and Trichoderma harzianum were subcultured and preserved in dishes on potato dextrose agar (PDA) for 18 months. The preservation was performed by treating conidia obtained from cultures. Conidia were subjected to three different methods of preservation: a) freeze-drying b) soil-sand and c) filter paper. A long-term maintenance is essential for detailed studies of the species. The viability and purity of the fungal species were monitored after 2 month -2 years storage at $10^{\circ} \mathrm{C}$. In preparing the suspension of spores or conidia, Triton X-100 and skim milk were used as stabilizer, and cryoprotectant respectively. The lyophilization method was favorable but destructible compared with the drying method on filter paper, resulting in severe damage to fungal materials which it was reflected in long germinating terms. The method of preservation in soil-sand showed lower viabilities. It is concluded that the freeze-drying method is a simple and reliable method for long-term preservation of the species studied.

Keywords: Viability, preservation, freeze-drying and filamentous fungi. 


\section{Introducción}

La calidad y cantidad de inóculo utilizado en los cultivos microbiológicos es uno de los factores más importantes en el desarrollo óptimo de estos. En el caso de los cultivos de hongos filamentosos, las características del inóculo influyen tanto en el tiempo de proceso como en la morfología desarrollada por el cultivo y en su productividad (Braun y Vecht 1991). El interés de los hongos filamentosos radica en la producción de metabolitos primarios (celulasas, lipasas, quitinasas, xilinasas, etc) y secundarios (aromas) y también como agentes biológicos de control. Con el propósito de estandarizar los procesos de producción de estos metabolitos es práctica común el uso de cepas preservadas en el desarrollo del inóculo primario. Muchos métodos se han desarrollado para la preservación de aislamientos fúngicos. De acuerdo a Gallo, Guimaraes, Momesso \& Pupo (2008), los métodos de preservación se dividen en dos grupos, en función al metabolismo continuo o suspendido del hongo. Los métodos que permiten el metabolismo continuo incluyen su almacenamiento en agua estéril, subcultivos en agar, almacenamiento en frio de $5-8^{\circ} \mathrm{C}$ y congelamiento a $-20^{\circ} \mathrm{C}$. Aunque el almacenamiento en agua estéril es un método sencillo y económico de preservación, provoca disminución en la velocidad de esporulación del hongo y cambios en sus características morfológicas (Borman, Szekely, Campbell y Johnson 2006). Los subcultivos de los aislamientos en agar es un método rutinario utilizado para preservar hongos debido a su simplicidad, pero el método puede volverse laborioso para un número grande de especies, presentando un mayor riesgo a contaminación y los cambios genéticos y fisiológicos no se pueden evitar (Kirsop y Doyle 1991). El almacenamiento en frio y la congelación han funcionado en preservar los aislamientos por períodos prolongados, pero aun estos métodos han demostrado ser inadecuados debido a las variabilidades biológicas de algunos microorganismos (Braun y Vecht 1991). Para propósitos de estudio, es importante mantener la unidad activa en un estado de dormancia de segura y fácil activación. La prolongación de la sobrevivencia de las conidias consiste en detener su germinación y reducir en lo posible su metabolismo. Una posibilidad para la estabilización es la deshidratación. El objetivo del presente trabajo fue la preservación de especies fúngicas por tres diferentes métodos comúnmente utilizados. La liofilización está entre las técnicas más utilizadas en la preservación de alimentos, productos agrícolas y fármacos (Ciurzynska y Lenart, 2011). Los materiales biológicos son especialmente sensibles a los tratamientos térmicos de deshidratación, sufriendo daños irreversibles (Ryan $\&$ Smith 2007). Para reducir estos efectos adversos, se adicionaron substancias protectoras a las muestras antes de procesarse. La sustancia protectora utilizada en el presente trabajo consistió de leche descremada (LD) y Tritón X-100. Se escogió LD debido sus características como agente emulsificador y estabilizador de membranas durante la criopreservación (Lalaymia, Cranenbrouck \& Declerck, 2014). El Tritón 
X-100 actúa como un excelente agente aglomerante e incrementa la viscosidad. Las tres tecnologías utilizadas se compararon en cuanto a su influencia en la viabilidad, velocidad de germinación de las conidias e integridad en la morfología de las colonias.

\section{Materiales y métodos}

\section{Microorganismos}

Se seleccionaron siete cepas de hongos filamentosos de los géneros y especies siguientes las cuales fueron donadas por la Universidad de Tecnologías Alimenticias de Plovdiv, Bulgaria y el Instituto de Ciencias Agrícolas de la Universidad Autónoma de Baja California. Las cepas se identificaron como Aspergillus niger, Aspergillus awamori, Aspergillus oryzae, Aspergillus flavus, Penicillium chrysogenum, Penicillium sp., y Trichoderma harzianum. Los cultivos se mantuvieron en placas en medio Agar Dextrosa Papa (ADP) a $4^{\circ} \mathrm{C}$, subcultivando periódicamente por 18 meses. La purificación e identificación de los hongos se hizo por la técnica de diluciones en agua destilada estéril hasta la dilución $10^{-7} ; 1 \mathrm{ml}$ de las suspensiones deseadas $\left(10^{-4}\right.$ y $\left.10^{-5}\right)$ fueron transferidos a placas por duplicado en medio ADP e incubados a $28 \pm 1^{\circ} \mathrm{C}$. Una vez que las cepas desarrollaron se compararon sus características morfológicas con claves taxonómicas para su identificación (Loustau-Gómez De Membrillo, 1950; Rifai, 1969). Se observó el crecimiento de las cepas en medio Agar Extracto de Malta (AEM) para el estudio de las estructuras fungales. Las observaciones al microscopio se realizaron por tinción de las estructuras usando azul de lactofenol como colorante. Se preservó la tinción sellando el cubreobjetos con barniz transparente (Leck, 1999).

\section{Métodos de Preservación}

Los siete microorganismos fueron cultivados por separado en cajas Petri conteniendo medio ADP de $15-20$ días a $28 \pm 1^{\circ} \mathrm{C}$ en la oscuridad para la producción de micelio y conidias necesarios en los diferentes métodos de preservación. Posteriormente los cultivos se preservaron por tres diferentes métodos: (1) En soporte suelo orgánico-arena; (2) Liofilizados y (3) Deshidratados y preservados en papel filtro, almacenando los tres a $10^{\circ} \mathrm{C}$. En los primeros dos métodos el micelio y las conidias de cada cultivo se removieron de la superficie del agar por raspado con una espátula estéril y preparando una suspensión de esporas. Para el cepario en suelo-arena, la suspensión de esporas se preparó con agua destilada estéril con Tritón X-100 (0.1\% v/v). En el caso del método de liofilización el material micelial y las conidias se suspendieron en una solución crioprotectora ( $20 \%$ leche descremada, $2 \%$ sacarosa y $0.1 \%$ Tritón X-100). 
Preservación en Suelo-Arena. Se preparó una mezcla de suelo orgánico y arena malla No. 35 en una relación $1: 3 \mathrm{v} / \mathrm{v}$ y se esterilizó con vapor a $120^{\circ} \mathrm{C}$ por dos horas. La mezcla de suelo se dejó al ambiente por 24 horas para permitir la germinación de esporas no eliminadas y se repitió el proceso de esterilización. A las 24 horas se rellenaron con esta mezcla tubos de vidrio de 15 x $100 \mathrm{~mm}$ con tapón de rosca a $2 / 3$ partes de capacidad y se esterilizaron con vapor a $120^{\circ} \mathrm{C}$ por dos horas y se inocularon con suspensión de esporas a partir de cultivos puros. Las suspensiones de esporas se prepararon removiendo el micelio y conidias de la superficie del agar por raspado con una espátula estéril, utilizando agua destilada estéril con Tritón X-100 $(0.1 \% \mathrm{v} / \mathrm{v})$. El volumen de suspensión utilizado fue de $2 \mathrm{ml}$ por tubo inoculado (Windels, Burnes \& Kommendahl, 1988). Se siguió el mismo procedimiento para cada microorganismo bajo condiciones de asepsia (Figura 1a y b).
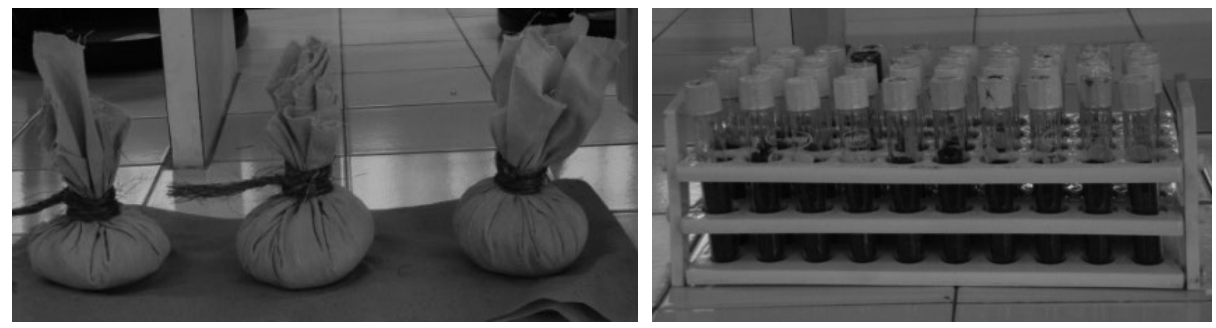

Fig 1. Cepario Suelo-arena. (a) Sustrato estéril (b) Preparación sustrato-suspensión de esporas

Preservación por liofilización. En el caso del método de liofilización el material micelial y las conidias se removieron de las cajas como se describió en el método anterior. Se suspendieron en una solución crioprotectora (20\% leche descremada, $2 \%$ sacarosa y $0.1 \%$ Tritón $\mathrm{X}-100$ ) y se llevaron a viales de $4 \mathrm{ml}$. Los viales con la suspensión fueron pre-congelados a $-30^{\circ} \mathrm{C}$ por $8 \mathrm{~h}$ y liofilizados en un equipo FreeZone Triad (Labconco) por 48 horas a $-25^{\circ} \mathrm{C}$ (Toegel, Salar-Behzadi, HoraczekClausen y Vierrnstein, 2010) (Figura 2).
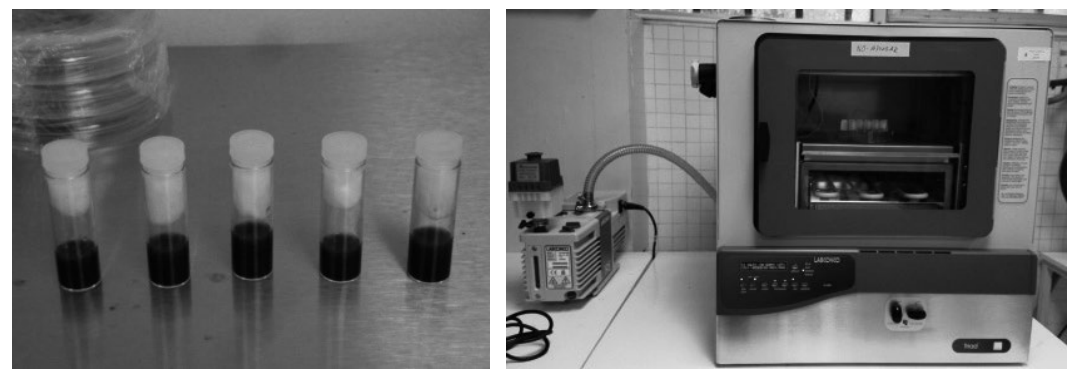

Fig 2. Liofilización de esporas. (a) Viales con suspensión de esporas para congelamiento (b) Proceso de liofilizado 
Preservación en papel filtro. El método utilizado fue una modificación del método desarrollado por Fong, Anuar, Lim, Tham y Sanderson (2000). Trozos de papel filtro Whatman No.1 de $1 \times 1 \mathrm{~cm}$ fueron envueltos en papel aluminio, esterilizados en una autoclave por $60 \mathrm{~min}$ a $121^{\circ} \mathrm{C}$ y posteriormente secados en horno a $100^{\circ} \mathrm{C}$ por 3 horas. Se colocaron cubriendo la superficie de placas con medio ADP. Micelio de 10 días de crecimiento fue transferido a los trozos de papel filtro por medio de aguja de disección. Cuando los cuadros de papel filtro en la placa fueron completamente cubiertos por micelio y esporas del hongo, se retiraron y separaron del medio PDA utilizando pinzas estériles. La mayoría de las especies de hongos tardaron más de 5 días para crecer sobre el papel filtro. El papel filtro con el micelio se colocó en placa estéril y llevada a la incubadora hasta su secado (20-25 días). Una vez que las transferencias en papel secaron, se colocaron de 3 a 4 piezas de papel filtro en sobres de papel celofán. Los sobres resultantes de cada especie se colocaron en bolsas de plástico estériles y rotuladas. Todas las bolsas se colocaron en un contenedor de plástico y se almacenaron a $10^{\circ} \mathrm{C}$. Figura 3.
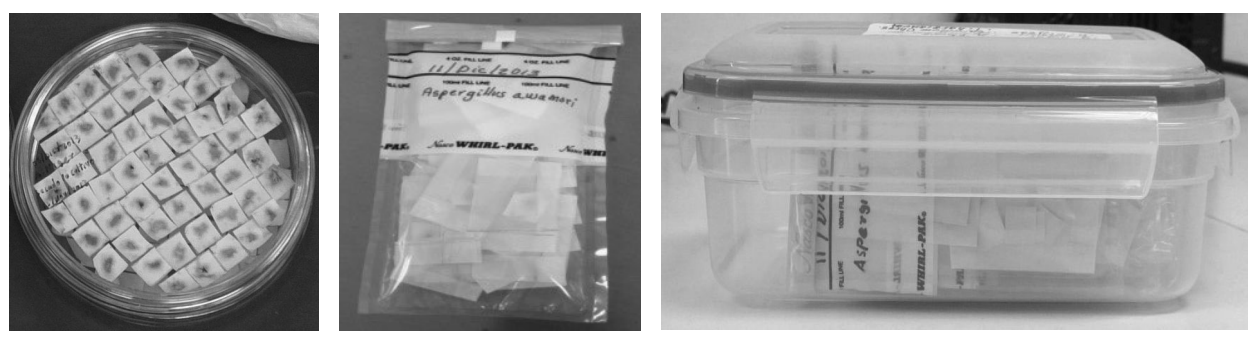

Fig 3. Cepario en papel filtro. (a) Inóculo en placa, (b) Bolsa estéril con sobres de papel celofán conteniendo papel filtro con esporas, (c) Almacenamiento de bolsas en contenedor de plástico.

\section{Pruebas de Viabilidad}

Las pruebas de viabilidad se realizaron en muestras almacenadas en un período de 2 meses a 2 años. Para los aislamientos en Suelo-arena la prueba de viabilidad se realizó aproximadamente a los 2 años de almacenamiento a $10^{\circ} \mathrm{C}$. La prueba se realizó salpicando unas cuantas partículas de suelo-arena en placas con medio PDA. Si el primer intento no tuvo éxito, entonces unas cuantas partículas de suelo arena se transfirieron en tubos de ensaye con $5 \mathrm{ml}$ de caldo dextrosa papa (CDP) dejando incubar de 24 a 48 horas a $28 \pm 1{ }^{\circ} \mathrm{C}$ con agitación orbital. Se observó la germinación colocando una gota del cultivo en un portaobjeto y tiñendo con azul de lactofenol en un microscopio (Iroscope MG-30PL) a 400X. Se consideraron conidias germinadas las que presentaron tubos germinados más largos que su diámetro. También se utilizó la técnica de cultivo de esporas en portaobjeto. En una placa con papel filtro Whatman húmedo se colocó un portaobjeto y se llevó a 
esterilizar a $121^{\circ} \mathrm{C}, 15$ min y se colocó una película delgada de medio PDA. Sobre este portaobjeto se colocaron $50 \mu \mathrm{l}$ de medio con la suspensión de esporas y se dejó incubar a temperatura ambiente $\left(26^{\circ} \mathrm{C}\right)$ con períodos alternos de luz oscuridad en un período de 24-48 hrs. El mismo procedimiento se utilizó para el cepario en papel filtro, y se determinó la viabilidad para 4 meses de almacenamiento a $10^{\circ} \mathrm{C}$. En el caso de las cepas preservadas por liofilización, una fracción del material liofilizado se re-suspendió en medio estéril CDP. A partir de una dilución de conidias de concentración conocida se sembraron 5 alícuotas de $100 \mu \mathrm{l}$ en placas con medio PDA. Se incubaron a $28^{\circ} \mathrm{C}$ durante 24 horas (Figura 4). El porcentaje de viabilidad se determinó contando las esporas germinadas con respecto al total de esporas presentes por campo en un total de 5 campos por microorganismo preservado (Oliveira, Pereira, Bento y Baptista, 2011).
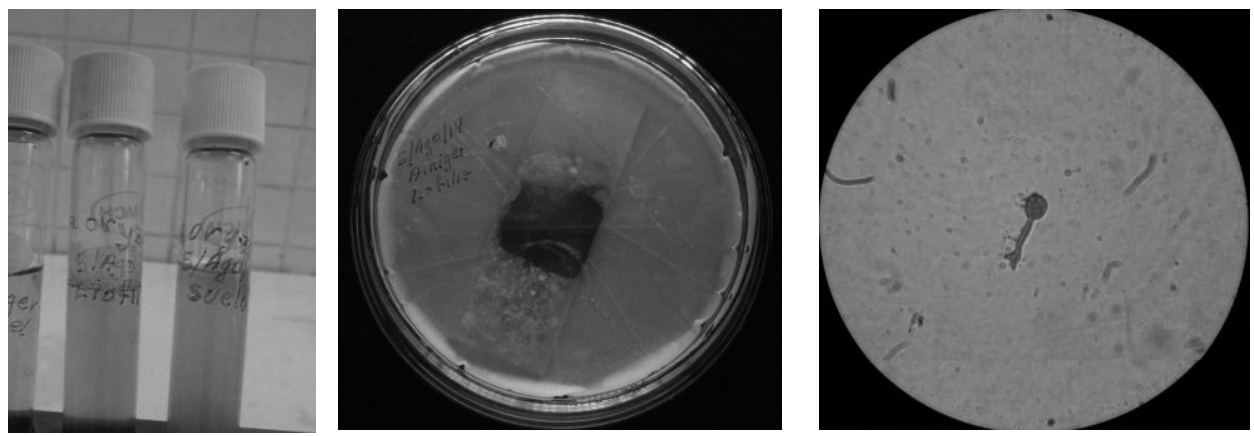

Fig 4. Pruebas de viabilidad en (a) CDP con 48 horas de incubación, (b) Portaobjeto en placa con medio ADP con 48 horas de incubación, (c) Espora germinada con tinción azul de lactofenol observada al microscopio, magnitud 100X.

\section{Resultados y discusión}

Las pruebas de viabilidad se hicieron a las 48 horas después de la reactivación de las conidias en CDP y ADP y los resultados fueron cercanos al 100\% de germinación de las esporas observadas al microscopio de luz para la mayoría de los aislamientos reactivados en ADP. El crecimiento de Aspergillus awamori fue más lento durante las 48 horas de incubación en los cultivos de reactivación tanto en ADP como en CDP. La apariencia de las colonias y la morfología de todas las especies almacenadas correspondieron a las claves taxonómicas consultadas, independientemente del tiempo de almacenamiento. Se encontró que con el incremento del tiempo de almacenamiento, el porcentaje de producción de colonias en suelo-arena disminuyó (Cuadro1). Las viabilidades promedio de los 7 aislamientos por método de preservación de liofilizado, en soporte papel y suelo- 
arena fueron $74.3 \%, 85.9 \%$ y $33.4 \%$ respectivamente. De los resultados se observa para aislamientos de hongos filamentosos una mayor reactivación del material conidial en el medio nutritivo sólido (ADP), comparado con el medio nutritivo líquido (CDP). Probablemente debido a la pobre dispersión celular en el medio líquido por la formación de aglomerados celulares.

Cuadro 1. Porcentajes de viabilidad de 7 diferentes hongos filamentosos preservados por liofilización, sustrato suelo-arena y en papel filtro a $10^{\circ} \mathrm{C}$

\begin{tabular}{|l|c|c|c|c|c|c|}
\hline \multirow{2}{*}{ Microorganismos } & \multicolumn{2}{|c|}{$\begin{array}{c}\text { Liofilizado } \\
\text { (10 meses) }\end{array}$} & \multicolumn{2}{c|}{$\begin{array}{c}\text { Papel filtro } \\
\text { (8 meses) }\end{array}$} & \multicolumn{2}{c|}{$\begin{array}{c}\text { Suelo-arena } \\
\text { (24 meses) }\end{array}$} \\
\cline { 2 - 8 } & CDP & ADP & CDP & ADP & CDP & ADP \\
\hline Aspergillus awamory & 50.0 & 100.0 & 40.0 & 1.0 & 10.0 & 10.0 \\
\hline Aspergillus flavus & 30.0 & 100.0 & 50.0 & 100.0 & 12.0 & 30.0 \\
\hline Aspergillus niger & 25.0 & 100.0 & 50.0 & 100.0 & 10.0 & 20.0 \\
\hline Aspergillus oryzae & 20.0 & 100.0 & 25.0 & 100.0 & 50.0 & 0.8 \\
\hline Penicillium chrysogenum & 50.0 & 100.0 & 25.0 & 100.0 & 4.0 & 80.0 \\
\hline Penicillium sp. & 60.0 & 100.0 & 10.0 & 100.0 & 20.0 & 80.0 \\
\hline Trichoderma harzianum & 25.0 & 10.0 & 80.0 & 100.0 & 25.0 & 20.0 \\
\hline $\begin{array}{l}\text { *La viabilidad se determinó para una sola muestra de cada aislamiento almacenado de los } \\
\text { diferentes métodos de preservación utilizados. }\end{array}$ \\
\hline
\end{tabular}

\section{Conclusiones}

La mayoría de los aislamientos usados en este estudio fueron preservados satisfactoriamente en los tres métodos utilizados. La cantidad de inóculo de la muestra tomada puede afectar la habilidad para detectar propágulos viables de los hongos. Pruebas iniciales arrojaron bajas sobrevivencias con altas diluciones de los aislamientos, pero cuando las pruebas se repitieron usando una mayor concentración de esporas, las viabilidades se incrementaron. Se recomienda el uso de medios de cultivo preparados artesanalmente de productos naturales en el proceso de reactivación de los aislamientos. 


\section{Referencias}

Borman, A., Szekely, A., Campbell, C. \& Johnson, E. (2006). Evaluation of viability of pathogenic filamentous fungi after prolonged storage in sterile water and review of recent published studies on storage methods. Mycopathologia, 161 (6), 361-68.

DOI: $10.1007 / \mathrm{s} 11046-006-0023-\mathrm{z}$

Braun, S. \& Vecht-Lifshitz, S. (1991). Mycelial morphology and metabolite production. Trends in Biotechnology, 9 (1), 63-68. DOI:10.1016/01677799(91)90020-I

Ciurzynska, A. \& Lenart, A. (2011), Freeze-Drying - Application in Food Processing and Biotechnology - A Review. Polish Journal of Food and Nutrition Sciences, 61 (3), 165-71. DOI: 10.2478/v10222-011-0017-5

Fong, Y.K., Anuar, S., Lim, H.P., Tham, F.Y. \& Sanderson, F.R. (2000). A modified filter paper technique for long-term preservation of some fungal cultures, Mycologist, 14 (3), 127-130. DOI:10.1016/S0269-915X(00)80090-7

Gallo, M. B., Guimaraes, D. O., Momesso, L. D. \& Pupo, M. T. (2008 ). Natural products from endophytic fungi. In Microbial Biotechnology. New India Publishing Agency, New Delhi, 139-168.

Kirsop, B. \& Doyle, A. (Eds.). (1991). Maintenance of microorganisms and cultured cells - a manual of laboratory methods, p. 17-18. London: Academic Press. http://tocs.ulb.tu-darmstadt.de/18929079.pdf

Lalaymia, I., Cranenbrouck, S. \& Declerck, S. (2014), Maintenance and preservation of ectomycorrhizal and arbuscular mycorrhizal fungi, Review, Mycorrhiza, 24 (5), 323-337. DOI: 10.1007/s00572-013-0541-8.

Leck, A. (1999). Preparation of lactophenol cotton blue slide mounts. Community Eye Health, 12 (30), 24. http://www.ncbi.nlm.nih.gov/pmc/articles/ PMC1706009/

Loustau-Gómez de Membrillera, J. (1950). Clave determinativa de las especies del género Penicillium. https://digitum.um.es/xmlui/ bitstream/10201/6407/1/N\%2017\%20\%20Clave\%20determinativa\%20 de\%20las\%20especies\%20del\%20genero\%20Penicillium.pdf

Loustau-Gómez de Membrillera, J. (1951). Clave determinativa de las especies del género Aspergillus. https://digitum.um.es/jspui/bitstream/10201/6483/1/N\%20 $3 \% 20$ Clave $\% 20$ determinativa $\% 20$ de $\% 201$ as $\% 20$ especies $\% 20$ de $1 \% 20$ 
genero\%20Aspergillus.pdf

Oliveira, I., Pereira, J.A., Bento, A. \& Baptista, P. (2011). Viability of Beauveria bassiana isolates after storage under several preservation methods. Annals of Microbiology, 61(2), 339-344. DOI: 10.1007/s13213-010-0147-8

Rifai, M. A. (1969). A revision of the genus Trichoderma. Mycological Papers, 116, p.1-56. http://www.mycobank.org/BioloMICS.aspx?Link=T\&TableKey= $14682616000000061 \&$ Rec $=2936 \&$ Fields $=$ All

Ryan, M. J. \& Smith, D. (2007). Cryopreservation and Freeze-Drying of fungi employing centrifugal and shelf Freeze-Drying. In Cryopreservation and Freeze-Drying Protocols, p. 127-40. Humana Press. DOI: 10.1007/978-159745-362-2_9

Toegel, S., Salar-Behzadi, S., Horaczek-Clausen A. Viernstein, H. (2010). Preservation of aerial conidia and biomasses from entomopathogeni fungi Beauveria brongniartii and Metarhizium anisopliae during lyophilization. Journal of Invertebrate Pathology, 105 (1), 16-23. DOI: 10.1016/j. jip.2010.05.004

Windels, C.E., Burnes, P. M., \& Kommendahl, T. (1988). Five-year preservation of Fusarium species on silica gel and soil. Phytopathology, 78 (1), 107109. https://www.apsnet.org/publications/phytopathology/backissues/ Documents/1988Articles/Phyto78n01_107.pdf 